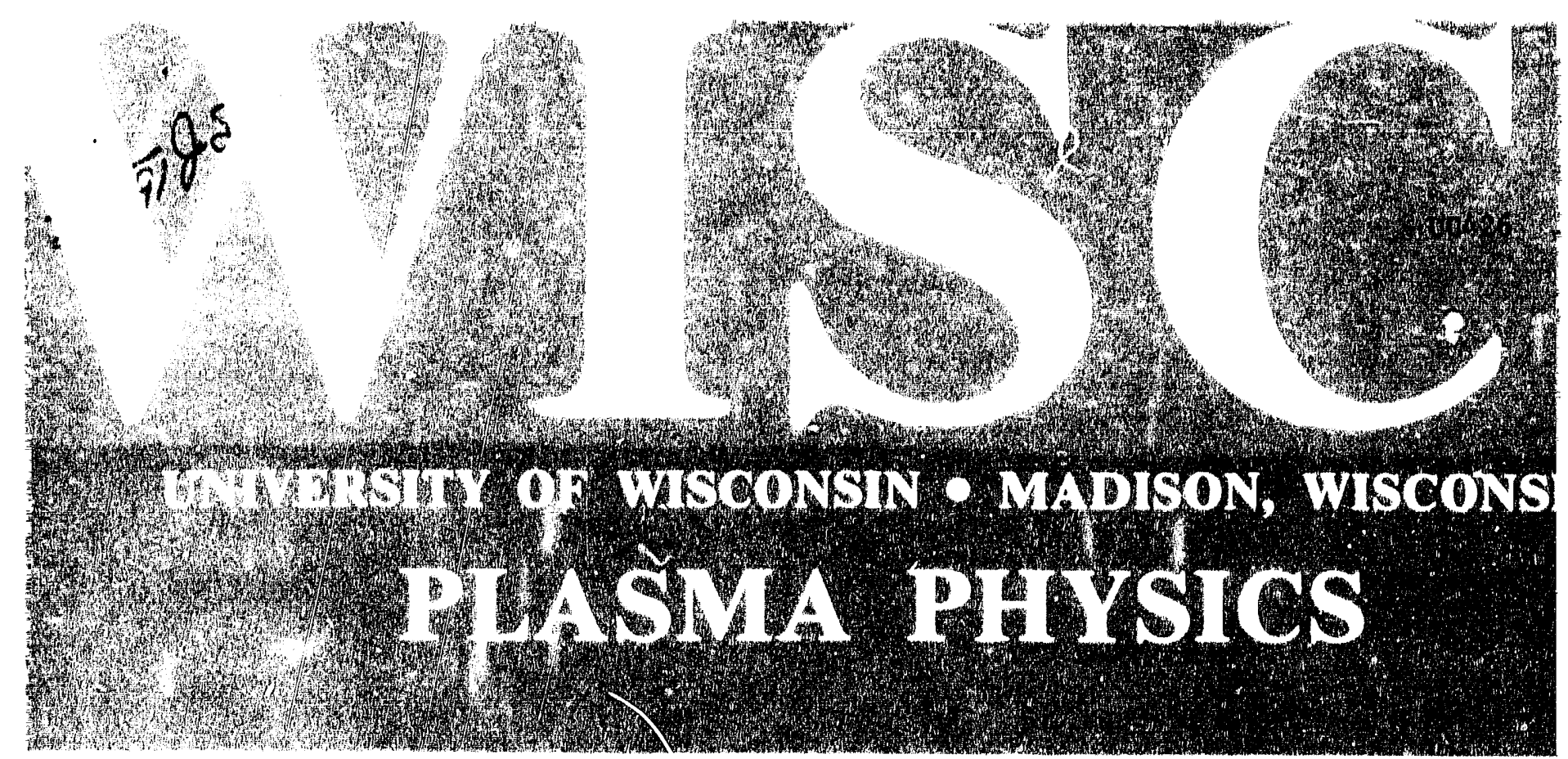



W. Shon and J.l. Sirrot






\section{NOTICE}

This report was prepared as an account of work sponsored by an agency of the United States Government. Neither the United States nor any agency thereof, nor any of thelr employees, makes any warranty, expressed or implled, or assumes any legal liablility or responsibility for any third party's use or the resuits of such use of any information, apparatus, product or process disclosed in this report, or represents that its use by such third party would not infringe privately owned rights.

Printed in the United States of Amerlca Available from

National Technical Information Service

U.S. Department of Commerce

5285 Port Royal Road

Springfield, VA 22161

NTIS Price codes

Printed copy: $\mathrm{A} 03$

Microfiche copy: A0I 
$\mathrm{DOE} / \mathrm{ER} / 53198--166$

\title{
Modified Polynomial Function Model for Reversed Field Pinches
}

\author{
W. Shen and J. C. Sprott \\ Department of Physics \\ University of Wisconsin, Madison, WI 53706
}

\begin{abstract}
A generalized analytic representation for the magnetic field and current density profiles in a reversed field pinch is proposed. These profiles have zero current density at the wall and finite plasma pressure. The profiles are characterized by two free parameters here taken to be the field reversal parameter $(F)$ and pinch parameter $(\Theta)$. From the profiles, many useful quantities such as magnetic energy, beta, inductance, resistance and ohmic input power are calculated. These quantities provide a basis for analyzing experimental data and performing electrical circuit modeling of RFP discharges.
\end{abstract}

PACS numbers: $52.55 . \mathrm{HC} \quad 52.55 \mathrm{Dy} \quad 52.30 \mathrm{Bt} \quad 52.65+\mathrm{z}$

\section{Introduction}

The magnetic field and current density profiles in a reversed field pinch (RFP) appear to relax toward what presumably represents a minimum energy state subject to certain constraints. If the constraints are constant toroidal magnetic flux and total magnetic helicity, the solutions for an axisymmetric, pressureless, cylindrical plasma are ${ }^{1}$

$$
B_{\phi}=B_{\phi}(0) J_{0}(2 \Theta r / a)
$$




$$
\begin{gathered}
B_{\theta}=B_{\phi}(0) J_{1}(2 \Theta r / a) \\
j_{\phi}=2 \Theta B_{\phi}(0) J_{0}(2 \Theta r / a) / \mu_{0} a \\
j_{\theta}=2 \Theta B_{\phi}(0) J_{1}(2 \Theta r / a) / \mu_{0} a
\end{gathered}
$$

In the equations above, a coordinate system $(r, \theta, \phi)$ is used in anticipation of applying the results to a toroid with large aspect ratio $\left(R_{0} / a\right)$ in which the toroidal circumference $2 \pi R_{0}$ replaces the length of the cylinder. The poloidal $(\theta)$ and toroidal $(\phi)$ fields vary with radius according to the Bessel function of zero and first order respectively, and hence this model is referred to as the Bessel function model (BFM). In the BFM a single parameter $(\Theta)$ uniquely determines the profile shape. Note that in the BFM the current is everywhere parallel to the field as required for a zero-pressure plasma in equilibrium and that the ratio $\mathbf{j} / \mathbf{B}$ is independent of radius:

$$
\lambda=\mu_{0} \mathbf{j} / \mathbf{B}=2 \Theta / \mathbf{a}
$$

A difficulty with the BFM is that it predicts a non-zero current density at the wall $(r=a)$ whereas experiment $2-5$ shows a vanishing current density as $r$ approaches a. For this reason, a variation of the $B F M$, called the modified Bessel function model (MBFM) is often used 6,7 in which $\lambda$ is taken constant for $0<r<b$ and is assumed thereafter to fall linearly to zero at $r=a$. The MBFM thus has two parameters $(\Theta$ and $b)$ and agrees better with experiment than the BFM. The best fit to experiment is found for b/a about 0.7 .

The MBFM still ignores plasma pressure which would produce a component of current perpendicular to $\mathbf{B}$ and requires a numerical evaluation of $\nabla \times \mathbf{B}=\lambda \mathbf{B}$ in order determine the field and current density profiles. For these reasons a model was proposed 8 in which the fields are expressed as polynomial functions of radius with enough terms kept in the polynomials to ensure that $\nabla \times \mathbf{B}=\mu_{0} \mathbf{j}, \mathbf{j}(\mathrm{a})$ $=0, \lambda^{\prime}(0)=0$ and $\lambda^{\prime \prime}(0)=0$. This polynomial function model (PFM) 
shares with the BFM the property of having a single parameter that specifies the shape of the fields, but unlike the other models, it has a perpendicular diamagnesic current whose value is consistent with the beta values observed in experiment. The expressions for the fields in the PFM are

$$
\begin{gathered}
B_{\phi}=B_{\phi}(0)\left[1-\Theta_{0}^{2}(r / a)^{2}+\Theta_{0}^{2}(r / a)^{4} / 2\right] \\
B_{\theta}=B_{\phi}(0) \Theta_{0}(r / a)\left[1-\Theta_{0}^{2}(r / a)^{2} / 2+\left(\Theta_{0}^{2}-1\right)(r / a)^{4} / 3\right] \\
j_{\phi}=2 B_{\phi}(0) \Theta_{0}\left[1-\Theta_{0}^{2}(r / a)^{2}+\left(\Theta_{0}^{2}-1\right)(r / a)^{4}\right] / \mu_{0} a \\
j_{\theta}=2 B_{\phi}(0) \Theta_{0}{ }^{2}(r / a)\left[1-(r / a)^{2}\right] / \mu_{0} a
\end{gathered}
$$

The parameter

$$
\Theta_{0}=\mu_{0} \mathrm{aj}_{\phi}(0) / 2 \mathrm{~B}_{\phi}(0)
$$

uniquely determines the profile shape for the PFM just as does $\Theta$ (or $\lambda$ ) in the BFM. This model has been used successfully to explain many of the properties of RFP discharges. $8-10$

When applied to specific RFP experiments, the PFM is too restrictive since it predicts a unique relationship $F(\Theta)$ in contrast to observations in which the plasma often deviates from such a unique curve. For this reason, a new model is here proposed, which, like the MBFM is characterized by two parameters (which we take to be $F$ and $\Theta)$ but which incorporates finite plasma pressure and fields whose variation can be expressed as analytic functions of radius. Since the functions are polynomials, we call this model the modified polynomial function model (MPFM). It generalizes the PFM in the same way that the MBFM generalizes the BFM, by introducing an additional parameter. The parameters are chosen to be those whose measurement is easiest ( $F$ and $\Theta$ ). From these analytic expressions, we proceed to calculate many useful formulas and then apply them 
to the analysis of experimental data and electrical circuit modeling of RFP discharges.

\section{Modified Polynomial Function Model}

For the MPFM, we start with Maxwell's equation $\nabla \times \mathbf{B}=\mu_{0} \mathbf{j}$ and apply the boundary conditions:

$$
\begin{aligned}
j(a) & =0 \\
\lambda^{\prime}(0) & =0
\end{aligned}
$$

Unlike in the PFM, however, we do not require $\lambda^{\prime \prime}(0)=0$. This means the same number of terms in the polynomial series for the fields as in the PFM can be used and still allow arbitrary beta values for each value of $\Theta$. The solutions for the fields are

$$
\begin{gathered}
B_{\phi}=B_{\phi}(0)\left[1-\Theta_{0}{ }^{2}(r / a)^{2}+\Theta_{0}^{2}(r / a)^{4} / 2\right] \\
B_{\theta}=B_{\phi}(0)(r / a)\left[\Theta_{0}-\left(2 \Theta_{0}-3 C\right)(r / a)^{2}+\left(\Theta_{0}-2 C\right)(r / a)^{4}\right] \\
j_{\phi}=2 B_{\phi}(0)\left[\Theta_{0}-2\left(2 \Theta_{0}-3 C\right)(r / a)^{2}+3\left(\Theta_{0}-2 C\right)(r / a)^{4}\right] / \mu_{0} a \\
j_{\theta}=2 B_{\phi}(0) \Theta_{0} 2(r / a)\left[1-(r / a)^{2}\right] / \mu_{0} a
\end{gathered}
$$

where

$$
C=B_{\theta}(a) / B_{\phi}(0)
$$

In an experiment, the easiest quantities to measure are $F$ and $\Theta$, given by

$$
F=B_{\phi}(a) /\left\langle B_{\phi}>\right.
$$




$$
\Theta=B_{\theta}(a) /\left\langle B_{\phi}\right\rangle
$$

where $\left\langle\mathrm{B}_{\phi}\right\rangle$ is the toroidal field averaged over the circular cross section

$$
<B_{\phi}>=\frac{2}{a} \int_{0}^{a} B_{\phi}(r) r d r
$$

which is related to the toroidal flux $\Phi$ by

$$
\Phi=\pi \mathrm{a}^{2}<\mathrm{B}_{\phi}>
$$

The parameters of the MPFM can thus be expressed in terms of $F$ and $\Theta$ as

$$
\begin{gathered}
\Theta_{0}=\sqrt{\frac{6-6 F}{3-2 F}} \\
C=\frac{\Theta}{3-2 F}
\end{gathered}
$$

from which it follows that

$$
\left.\mathrm{B}_{\phi}(0)=(3-2 F)<\mathrm{B}_{\phi}\right\rangle
$$

The radius at which the toroidal field reverses is independent of $\Theta$ and is given by

$$
r_{R}=a \sqrt{1-\sqrt{\frac{F}{3 F-3}}}
$$

The MPFM can also be used to calculate the normalized internal plasma self-inductance defined by 11 


$$
L_{i}=\frac{2 \int_{0}^{a} B_{\theta}{ }^{2} r d r}{a^{2} B_{\theta}^{2}(a)}
$$

In terms of $F$ and $\Theta, L_{i}$ is given by

$$
l_{i}=\frac{24 F^{2}-60 F+36+8 \Theta \sqrt{(6-6 F)(3-2 F)}+31 \Theta^{2}}{60 \Theta^{2}}
$$

which can be combined with the value of $\beta_{\theta}$ derived in section IV to obtain the asymmetry factor defined by ${ }^{12}$

$$
\Lambda=\beta_{\theta}+l_{i} / 2-1
$$

The asymmetry factor is a measure of the outward shift of the flux surfaces that results when the pinch is bent into a toroid. Experimentally, $\Lambda$ is found to be close to zero in RFP devices $13-16$. The result is an expression for $\Lambda$ in terms of only $F$ and $\Theta$ :

$$
\Lambda=\frac{48 F^{2}+132 F-180+8 \Theta \sqrt{(6-6 F)(3-2 F)}+31 \Theta^{2}}{120 \Theta^{2}}
$$

Contours of constant $\Lambda$ in $F-\Theta$ space are shown in Fig. 1.

\section{Experimental Comparisons}

The equilibrium magnetic field profiles have been measured on many RFP devices. ${ }^{2-5}$ On the MST device at the University of Wisconsin 17 , insertable magnetic probes with multiple coils measuring magnetic field in three orthogonal directions have been used to study the magnetic field evolution and the magneticfluctuation-induced transport. To compare with the MPFM, measurements of the magnetic field profile were made by moving 
the coils to different positions across the outer part of the plasma minor radius. The signals obtained from the coils were then processed through analog integrators to get the magnetic field at each position.

The probes consisted of six coils (2 for each orientation) made from copper wires and mounted on thin stainless steel tubing and inserted into either graphite or boron nitride shields with an outer diameter of $1.5 \mathrm{~cm}$. The shields protect the coils from overheating by the plasma heat flux. To minimize the error pickup field due to the plasma equilibrium outward shift, the probes were inserted horizontally.

All the data were taken at low plasma current $\left(l_{\phi} \sim 200 \mathrm{kA}\right)$ because the high current plasmas would generate a large heat flux and destroy the probes in a few shots. Even for the low-current cases the probes could only be inserted to about $15 \mathrm{~cm}$ from the wall (whose radius is at $a=52 \mathrm{~cm}$ ) since either the plasma was significantly perturbed or the probes could not survive the heat pulse.

The data were digitized with LeCroy 8210 digitizers at frequencies up to $200 \mathrm{kHz}$. The results shown in Fig. 2 were taken at the time of peak current and represent averages of several shots. The MPFM predictions are shown as solid curves and were calculated using the measured values of $F$ and $\Theta$, and thus contain no adjustable parameters.

A second point of comparison of the model with MST was made by Almagri 18 who measured the asymmetry factor $\Lambda$ using an array of sixteen coils at the surface of the wall at a single toroidal azimuth but distributed around the poloidal circumference. The poloir'n field at the wall was Fourier analyzed to obtain the $m=1$ component which is proportional to $\Lambda$. Fifty shots were taken over a range of $1.7<\Theta<$ $2.1(0>F>-0.37)$, and the inferred values of $\Lambda$ fell in the range $-0.16<\Lambda<0$. The values calculated by the MPFM were in agreement to within a standard deviation of $\delta \Lambda= \pm 0.02$. 


\section{Derived Quantities}

A crucial test of the model is whether the perpendicular diamagnetic currents are consistent according to $\mathbf{j} X \mathbf{B}=\nabla p$ with the values of plasma pressure observed experimentally. In order to test this prediction, we define a volume-averaged $\beta_{\theta}$ in the customary manner,

$$
\beta_{\theta}=2 \mu_{0}<p>/ B_{\theta}^{2}(a)
$$

and calculate its value as predicted by the MPFM:

$$
\beta_{\theta}=\frac{4 \mu_{0}}{a^{2}{ }_{\theta}^{2}(a)} \int_{0}^{a} \int_{r}^{a}\left(j_{\phi} B_{\theta}-j_{\theta} B_{\phi}\right) d r d r
$$

with the result

$$
\beta_{\theta}=1-\left(9-8 F-F^{2}\right) / 5 \Theta^{2}
$$

Thus each point in F- $\Theta$ space has associated with it a unique value of $\beta_{\theta}$ as indicated in Fig. 3. The predicted beta values are consistent with the range of values observed in RFP experiments. Any attempt to infer the value of beta from an experimental $F-\Theta$ curve is unlikely to be successful since small profile changes effectively mask any changes in beta. In fact it is fortuitous that the beta predicted by the model is positive; it could just have well been negative. In the case of MST, the predicted beta is about twice what is actually measured, although there is considerable uncertainty since the experimental profiles are not accurately known.

In similar fashion the total plasma energy can be calculated, 


$$
U_{p}=6 \pi^{2} R_{0} \int_{0}^{a} \int_{r}^{a}\left(j_{\phi} B_{\theta}-j_{\theta} B_{\phi}\right) d r r d r
$$

to give

$$
U_{p}=3 R_{0} \Phi^{2}\left[5 \Theta^{2}-9+8 F+F^{2}\right] / 10 \mu_{0} a^{2}
$$

Finally, the total magnetic energy is given by

$$
U_{m}=\frac{2 \pi^{2} R_{0}}{\mu_{0}} \int_{0}^{a} B^{2}(r) r d r
$$

which evaluates to

$$
U_{m}=\frac{R_{0} \Phi^{2}}{60 \mu_{0} a^{2}}\left[144-156 F+72 F^{2}+8 \Theta \sqrt{(6-6 F)(3-2 F)}+31 \Theta^{2}\right]
$$

Contours of constant $U_{m}$ normalized to $R_{o} \Phi^{2} / \mu_{o} a^{2}$ are plotted in $F$ $\Theta$ space in Fig. 4.

\section{Electrical Circuit Parameters}

As a starting point for calculating the electrical circuit parameters for an RFP we consider the energy balance equation,

$$
V_{\phi} I_{\phi}+V_{\theta} I_{\theta}=d U_{m} / d t+P_{o h}
$$

where the terms on the left-hand side can be thought of as the Poynting flux at the plasma surface produced respectively by the poloidal and toroidal field windings. This input power is distributed between increasing the stored magnetic energy and ohmic power dissipation by the plasma. Other losses such as that required to 
magnetize the space external to the shell that surrounds the plasma and resistive heating of the liner are considered part of the external circuit. The magnetic energy $U_{m}$ is taken to be a function of $I_{\phi}, I_{\theta}$ and $\Phi$, in terms of which $F$ and $\Theta$ can be expressed as

$$
\begin{gathered}
F=\mu_{0} a^{2} I_{\theta} / 2 R_{0} \Phi \\
\Theta=\mu_{0} a l_{\phi} / 2 \Phi
\end{gathered}
$$

Thus the rate of change of $U_{m}$ can be decomposed into three parts,

$$
\frac{d U_{m}}{d t}=\frac{\partial U_{m}}{\partial I_{\phi}} \frac{d l_{\phi}}{d t}+\frac{\partial U_{m}}{\partial I_{\theta}} \frac{d l_{\theta}}{d t}+\frac{\partial U_{m}}{\partial \Phi} \frac{d \Phi}{d t}
$$

Now we define a resistive voltage $V_{R}$ such that its product with the toroidal plasma current gives the ohmic input power,

$$
P_{o h}=V_{R} l_{\phi}=V_{\phi} l_{\phi}+V_{\theta} l_{\theta}-d U_{m} / d t
$$

and define three new parameters $L, M$ and $A$ such that

$$
V_{R}=V_{\phi}-L d l_{\phi} / d t+M d l_{\theta} / d t-A V_{\theta}
$$

where use has been made of the fact that $V_{\theta}=d \Phi / d t$. The three new parameters are given by

$$
\begin{gathered}
L=\frac{1}{I_{\phi}} \frac{\partial U_{m}}{\partial I_{\phi}} \\
M=-\frac{1}{I_{\phi}} \frac{\partial U_{m}}{\partial I_{\theta}} \\
A=\frac{1}{I_{\phi}} \frac{\partial U_{m}}{\partial \Phi}-\frac{l_{\theta}}{I_{\phi}}
\end{gathered}
$$


These are the circuit parameters that are required to predict the tehavior of an RFP when connected to an external circuit. Their evaluation from the MPFM is straightforward but tedious:

$$
\begin{gathered}
L=\frac{\mu_{0} R_{0}}{120}\left[31+\frac{4}{\Theta} \sqrt{(6-6 F)(3-2 F)}\right] \\
M=\frac{\mu_{0} a}{20 \Theta}\left[13-12 F+\frac{2 \Theta(5-4 F)}{\sqrt{(6-6 F)(3-2 F)}}\right] \\
I=\frac{R_{0}}{10 a}\left[\frac{2(6-5 F)}{\sqrt{(6-6 F)(3-2 F)}}+\frac{24-13 F}{\Theta}\right]-\frac{R_{0} F}{a \Theta}
\end{gathered}
$$

Tirese relations provide a practical means for calculating the resistive voltage and ohmic input power to an RFP plasma whenever $I_{\phi}, I_{\theta}$, and $\Phi$ are changing in time.

The p!asma resistance given by $R_{P}=V_{R} / I_{\phi}$ can also be calculated in terms of the plasma resistivity $\eta$, assuming negligible conductivity perpendicular to $\mathbf{B}$ using the relation

$$
I_{\phi}^{2} R_{p}=\int_{0}^{a} \frac{\eta(j \cdot B)^{2}}{B^{2}}-4 \pi^{2} R_{0} r d r
$$

If $\eta$ is constant in space, the integral above has a unique value for the MPFM frum which the resistance can be calculated as follows:

$$
R_{p}=\frac{4 \pi^{2} R_{0} \eta}{l_{\phi}^{2}} \int_{0}^{a} \frac{\left(j_{\phi} B_{\phi}+j_{\theta} B_{\theta}\right)^{2}}{B_{\phi}^{2}+B_{\theta}^{2}} r d r
$$

Unfortunately, the above expression seems not to have a simple analytic represeritation, but the value of $R_{p}$ normalized to $2 R_{0} \eta / a^{2}$ (often called the "screw-up factor") can be evaluated numericaliy. 
From its value, the average resistivity $\eta$ can be determined from experimentally measured quantities. Setting the measured value of $\eta$ to the Spitzer value (with $Z_{\text {eff }}=1$ ) gives the conductivity temperature. Alternately, if the temperature is independently known, the calculation allows one to calculate $Z_{\text {eff }}$ or the resistance anomaly. Note that the screw-up factor is not zero at $\Theta=0$ because of the non-uniform toroidal current density required to satisfy $j(a)$ $=0$.

The circuit parameters are complicated functions of both $F$ and $\Theta$. Their values hive been calculated versus $\Theta$ for various $\beta_{\theta}$ using the MPFM and the results plotted in Figs 5-8. The values have also been calculated along the F- $\Theta$ curve corresponding to the PFM, and for the range $1<\Theta<2$ are found to fit within $2 \%$ the simple functions:

$$
\begin{gathered}
L \cong(0.297+0.043 \Theta) \mu_{0} R_{0} \\
M \cong(0.222+0.246 \Theta) \mu_{0} a \\
A \cong(0.503+1.017 \Theta) R_{0} / a \\
R_{p} \cong 2(-0.685+2.816 \Theta) R_{0} \eta / a^{2}
\end{gathered}
$$

\section{Circuit Modeling}

An RFP plasma can be considered as a two-port electrical network in which the poloidal and toroidal field circuits are coupled nonlinearly through the plasma.6-8,19 In such a case the variables are $V_{\phi}, I_{\phi}, V_{\theta}$ (or $\Phi$ ) and $I_{\theta}$. Thus four equations are needed to solve the system. Two of the equations are provided by the external electrical circuits connected to the windings. The third comes from the definition of $V_{R}$ and some assumption about the plasma resistivity, and the fourth can be deduced from the plasma energy 
balance equation,

$$
d U_{p} / d t=I_{\phi} V_{R}-U_{p} / \tau
$$

with some assumption about the functional variation of energy confinement time $\tau$ with the other parameters. To these one must add equations for any additional external electrical circuit components connected to the field windings. For any given case, the equations can be reduced to a system of first-order, ordinary, initial-value, differential equations which can be solved by any of the standard methods.

As a specific example, consider a case in which $V_{R}$ and $\tau$ are constants, the poloidal-field circuit is connected at $t=0$ to $a$ capacitor bank $C_{P}$ charged to voltage $V_{\phi}(0)$ and the toroidal-field circuit is connected to a series inductor $L_{T}$, resistor $R_{T}$ and voltage source $V_{T}$ in which an initial current $I_{\theta}(0)$ is flowing. The system of equations to be solved would then be:

$$
\begin{gathered}
\frac{d V_{\phi}}{d t}=-\frac{I_{\phi}}{C_{P}} \\
\frac{d l_{\phi}}{d t}=\frac{1}{L}\left[V_{\phi}-V_{R}+M \frac{d l_{\theta}}{d t}-A \frac{d \Phi}{d t}\right] \\
\frac{d \Phi}{d t}=-L T \frac{d l_{\theta}}{d t}-R T I_{\theta}+V_{T} \\
\frac{d l_{\theta}}{d t}=\frac{\left(V_{T}-I_{\theta} R T\right)\left(54 \Phi-24 I_{\theta} L\right)+20 L\left(V_{R} I_{\phi}-U_{p} / \tau\right)-15\left(V_{\phi}-V_{R}\right) I_{\phi} R_{0} \mu_{0}}{15(A L T+M) I_{\phi} R_{O \mu_{0}}+6 I_{\theta} L\left(L_{C}-4 L T\right)+6 \Phi(9 L T+4 L)}
\end{gathered}
$$

where $L, A M$ and $U_{p}$ are given in terms of the four variables for the MPFM by formulas previously derived.

Although the above equations are written with time-derivatives on the right-hand side for simplicity, they can be evaluated in an order such that only the values of the variables need be known (for 
example: $V_{\phi}, I_{\theta}, \Phi$, and finally $\left.I_{\phi}\right)$. In addition to $V_{\phi}(0)$ and $I_{\theta}(0)$, two additional initial conditions must be specified. The usual initial conditions will be $\Theta=0$ [or $I_{\phi}(0)=0$ ] and $F=1$ [or $\Phi(0)=$ $\left.\mu_{0} a^{2} l_{\theta}(0) / 2 R_{0}\right]$, although there may be some difficulty with numerical instability if the solution is started exactly from such a vacuum condition.

\section{Vil. Time-Dependent Solutions}

The system of time-dependent equations above were solved for parameters typical of the MST device 17 as it currently operates. The device has a major radius of $R_{0}=1.5 \mathrm{~m}$ and a minor radius of $\mathrm{a}=$ $0.52 \mathrm{~m}$. The poloidal-field capacitor bank is represented by a oneturn equivalent capacitance of $32.4 \mathrm{~F}$, and it is typically charged initially to a one-turn equivalent voltage of $180 \mathrm{~V}$. The toroidalfield circuit is assumed to be imperfectly crowbarred through a single-turn equivalent inductance of $0.1 \mu \mathrm{H}$ with a resistance of 10 $\mu \Omega$ and a fixed externally applied voltage of $V_{T}=-2 \mathrm{~V}$. The plasma is assumed to have a resistance such that the resistive voltage $V_{R}$ is a constant $15 \mathrm{~V}$, and the energy confinement time $\tau$ is assumed to be a constant $1 \mathrm{~ms}$. The other initial conditions were chosen to be $\mathrm{I}_{\phi}=$ $0.1 \mathrm{~A}, \Phi=0.136 \mathrm{~Wb}$ and $\mathrm{I}_{\theta}=1.2 \mathrm{MA}$. The poloidal-field circuit is passively crosubarred when $V_{\phi}$ reaches zero. The resulting waveforms as shown in Fig. 9 agree well with typical MST discharges as reported elsewhere. ${ }^{17}$

\section{Linear Stability of the Circuit Equations}

The circuit equations of section VI consist of four, first-order, coupled, nonlinear, ordinary differential equetions. Such a system

presents the possibility of complex behavic $r$ including phenomena such as oscillations, instability, bifurcations and chaos. The 
equations have been subjected to a linear perturbation analysis about a time-independent equilibrium. To get a time-independent equilibrium solution, $C_{p}$ is set to infinity so that $V_{\phi}$ is constant. Each of the variables is assumed to be given by the superposition of a constant (zeroth-order) term and a small (first-order), complex, oscillatory perturbation, as for example,

$$
I_{\phi}=I_{\phi 0}+I_{\phi 1} e^{i \omega t}
$$

The zeroth-order terms lead to a set of equilibrium equations,

$$
\begin{gathered}
V_{\phi 0}=V_{R} \\
I_{\phi 0}=U_{p 0} / V_{R^{\tau}}^{\tau} \\
I_{\theta 0}=V_{T} / R_{T}
\end{gathered}
$$

in which the fourth variable, $\Phi_{0}$, can be chosen arbitrarily.

The first-order terms lead to an algebraic equation for the complex frequency $\omega$ of the form

$$
\begin{gathered}
-\left(20 M V_{R} \tau_{1}-3 I_{\theta 0} L_{T}-24 L \Phi_{0}\right) L_{c} \tau \omega \omega^{2} \\
+\left\{\left[20\left(\tau_{1}+\tau\right) M V_{R^{-}} 3 I_{\theta 0} L L_{c}-24 L \Phi_{0}\right] L_{c}+4\left(6 I_{\theta 0} L_{T}+5 L_{c} V_{R} \tau_{1}-27 L \Phi_{0}\right) R_{T} \tau\right\} i \omega \\
+4\left[6 I_{\theta 0} L L_{c}+5\left(\tau_{1}+\tau_{2}\right) A L_{c} V_{R^{2}}-27 L \Phi_{0}\right] R_{T}=0
\end{gathered}
$$

where several new variables have been defined:

$$
\begin{gathered}
\tau_{1}=\left.3 \mu_{0} R_{0}\right|_{\phi 0} / 4 V_{R} \\
\tau_{2}=3\left(2 L_{c}+9 L_{T}\right) L \Phi_{0} / 5\left(A L_{T}+M\right) L_{c} V_{R} \\
L_{c}=\mu_{0} a^{2} / 2 R_{0}
\end{gathered}
$$

$L_{c}$ is the internal inductance of the toroidal-field circuit in the 
absence of plasma. The above equation takes on a simpler form for the case of $R_{T}=0$ :

$$
i \omega \tau=\frac{6\left(F^{2}+8 F+5 \Theta^{2}-9\right)}{\frac{2 \Theta \Theta_{0}\left(4 \Theta_{0}+31 \Theta\right)\left[(F+4) L_{c}+(9-4 F) L_{T}\right]}{2 \Theta\left[(5-4 F) L_{c}+(6-5 F) L_{T}\right]+\Theta_{0}\left[(13-12 F) L_{c}+(24-23 F) L_{T}\right]}+15 \Theta^{2}}-1
$$

Although this equation is a complicated function of $L_{T}, F$ and $\Theta$, for the experimentally interesting range of parameters, it reduces approximately to

$$
i \omega \tau \cong \beta_{\theta} / 3-1
$$

Thus one can see that for the usual case of $\beta_{\theta} \ll 1$, perturbations exponentially damp in a time approximately equal to the energy confinement time $\tau$, and the equations have linearly stable solutions, at least so long as $V_{R}$ and $\tau$ are constant.

The analytical results above have been verified using a timedependent numerical calculation to observe the decay of a small $(5 \%)$ perturbation of $\Phi$ about its equilibrium value. Parameters were chosen to be representative of an MST discharge: $l_{\phi}=350 \mathrm{kA}, I_{\theta}=-$ $205 \mathrm{kA}, \Phi=0.10715 \mathrm{~Wb}, \Theta=1.6, \mathrm{~F}=-0.325, \beta_{\theta}=10 \%, \mathrm{~L}_{T}=0.1 \mu \mathrm{H}, \mathrm{V}_{\mathrm{R}}$ $=25 \mathrm{~V}$ and $\tau=1 \mathrm{~ms}$. With $R_{T}=0$, an exponential decay back to the equilibrium value is observed with a decay time within a few percent of the energy confinement time. With $R_{T}>0$, the perturbation still damps on the same time scale but with a real (oscillatory) part of frequency

$$
\omega_{R} \cong 2 \sqrt{\frac{R_{T}}{\tau L_{T}}}
$$

There are many other cases that could be examined in which, for example, $V_{R}$ and $\tau$ are functions of $I_{\phi}$ and $U_{p}$. A number of such cases have been studied numerically with a result that resembles that described above or perhaps a slow (>50 ms) growth of the 
perturbation. This comment is not meant to exclude the possibility of cases in which interesting oscillatory or chaotic phenomena occur, but the variety of possibilities is large, and some guidance is required from experiment or from further theoretical calculations.

\section{Conclusions}

The modified polynomial function model provides a reasonable representation of the magnetic fields in a reversed field pinch in terms of simple polynomial functions of the minor radius. The coefficients of the polynomial terms are functions only of the easily measured parameters $F$ and $\Theta$. The profiles agree well with experimental measurements on the MST device.

From the model profiles, analytic expressions have been obtained for such quantities as magnetic fiek: on axis, field reversal radius, total plasma and magnetic energies, poloidal beta and various electrical circuit parameters all in terms of $F$ and $\Theta$. The circuit parameters provide a means for calculating the ohmic input power, resistive voltage and bulk plasma resistance from experimentally measured quantities when the plasma is not in a steady state. The circuit parameters alsc allow one to predict experimental waveforms for an RFP plasma coupled through its poloidal and toroidal field windings to external electrical circuit components. The waveforms are found to agree well with those observed on the MST device.

The linear stability of the electrical circuit equations has been examined. For a range of conditions typical of experimental operation, the equations are stable, and perturbations to the equilibrium damp on the time scale of the plasma energy confinement time. Thus nonstationary behavior such as sawtooth oscillations do not appear to result from the model. 


\section{Acknowledgments}

This work was supported by the U. S. Department of Energy grant No. DE-FG02-85ER53198.

\section{References}

1. J. B. Taylor, Phys. Rev. Lett. 33, 1139 (1974).

2. H. A. B. Bodin and A. A. Newton, Nucl. Fusion 20, 1255 (1980).

3. R. J. La Haye, T. N. Carlstrom, R. R. Goforth, G. L. Jackson, M. J. Schaffer, T. Tamano, and P. L. Taylor, Phys. Fluids 27, 2576 (1984).

4. V. Antoni and S. Ortolani, Phys. Fluids 30, 1489 (1987).

5. J. S. Sarff, J. C. Sprott, and L. Turner, Phys. Fluids 30, 2155 (1987).

6. J. W. Johnson, Plasma Phys. 23, 187 (1981).

7. K. F. Schoenberg, R. F. Gribble, and J. A. Phillips, Nucl. Fusion 22, 1433 (1982).

8. J. C. Sprott, Phys. Fluids 31, 2266 (1988).

9. Y. Hirano, Y. Yagi, T. Shimada, K. Hattori, Y. Maejima, I. Hirota, Y. Kondoh, K Saito and S. Shiina, Proc. 13th Int. Conf. on Plasma Phys. and Conirolled Nuclear Fusion, IAEA, Washington (1990), paper IAEA$\mathrm{CN}-53 / \mathrm{C}-4-12$, to be published.

10. K. L. Sidikman, S. D. Schultz and P. W. Terry, Bull. Am. Phys. Soc 35, 1981 (1990). 
11. V. S. Mukhovatov and V. D. Shafranov, Nucl. Fusion 1, 605 (1971).

12. H. A. B. Bodin and A. A. Newton, Nucl. Fusion 20, 1255 (1980).

13. D. C. Robinson, Plasma Physics 11, 893 (1969).

14. H. A. B. Bodin, E. P. Butt, J. E. Crow, F. E. Irons, J. Junker, A. A. Newton and D. C. Robinson in Plasma Physics and Controlled Nuclear Fusion Research, IAEA: Vienna (Proc. 4th Int. Conf. Madison, 1971) 1, 225 (1971).

15. D. Baker, L. C. Burkhardt, J. N. DiMarco, P. R. Forman, A. Haberstitch, R. B. Howell, H. J. Karr, J. A. Phillips, A. E. Schofield in Plasma Physics and Controlled Nuclear Fusion Research, IAEA: Vienna (Proc. 5th Int. Conf. Tokyo, 1974) 3, 35 (1975).

16. A. Buffa, S. Costa, L. Fellin, G. Malesani, P. L. Mondino, G. F. Nalesso, S. Ortolani, G. Rostagni and A. Stella in Plasma Physics and Controlled Nuclear Fusion Research, IAEA: Vienna (Proc. 5th Int. Conf. Tokyo, 1974) 3, 431 (1975).

17. R. N. Dexter, D. W. Kerst, T. W. Lovell, S. C. Prager and J. C. Sprott, Fusion Technology, accepted for publication.

18. A. F. Almagri, University of Wisconsin Ph.D. Thesis (1990).

19. K. F. Schoenberg, R. F. Gribble, and D. A. Baker, J. Appl. Phys. 56, 2519 (1984). 


\section{Figure Captions}

1. Contours of constant asymmetry factor $\Lambda$ in $F-\Theta$ space as predicted by the modified polynomial function model.

2. Magnetic field profiles measured in MST (crosses) compared with the predictions of the modified polynomial function model (solid curves).

3. $F-\Theta$ plot $f(r$ the modified polynomial function model at various values of $\beta_{\theta}$ compared with the Bessel function model (BFM) and the polynomial function model (PFM).

4. Normalized contours of magnetic energy in F- $\Theta$ space as predicted by the modified polynomial function model.

5. Normalized self-inductance of an RFP plasma with fixed $\mathrm{I}_{\theta}$ and $\Phi$.

6. Normalized mutual inductance of an RFP plasma with fixed $I_{\phi}$ and $\Phi$

7. Normalized coupling coefficient of an RFP plasma with fixed $I_{\theta}$ and $I_{\phi}$.

8. Normalized resistance $\left(V_{R} / /_{\phi}\right)$ of an RFP plasma with a spatially independent parallel resistivity $\eta$.

9. Numerical solutions of the time-dependent MPFM circuit equations for a case resembling the MST experiment $\left(R_{0}=1.5 \mathrm{~m}, a=0.52 \mathrm{~m}, C_{p}\right.$ $=32.4 \mathrm{~F}, \mathrm{~L}_{\mathrm{T}}=0.1 \mu \mathrm{H}, \mathrm{R}_{\mathrm{T}}=10 \mu \Omega, \mathrm{V}_{\mathrm{T}}=-2 \mathrm{~V}, \mathrm{~V}_{\mathrm{R}}=15 \mathrm{~V}$ and $\left.\tau=1 \mathrm{~ms}\right)$. 


$$
r
$$




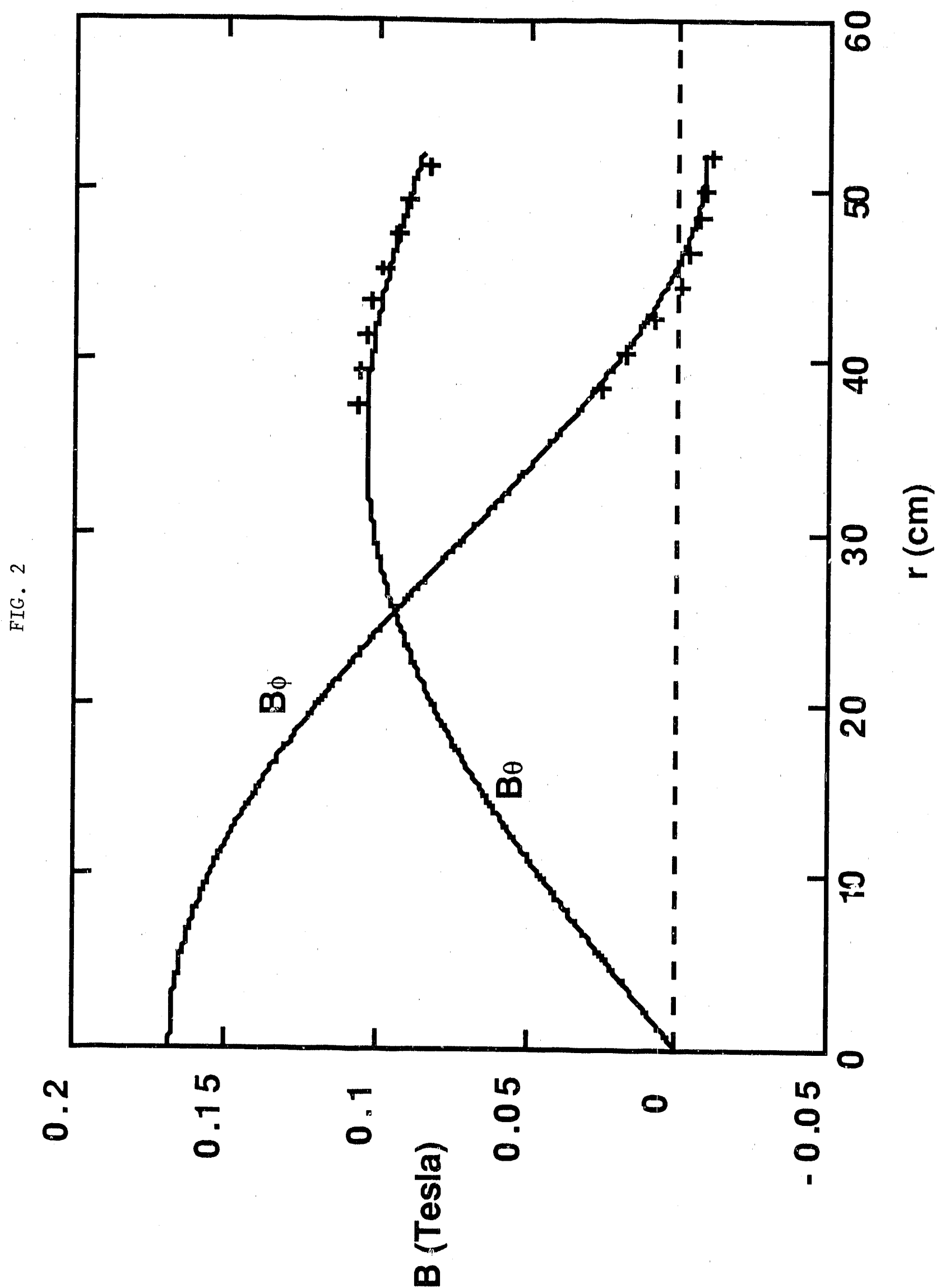




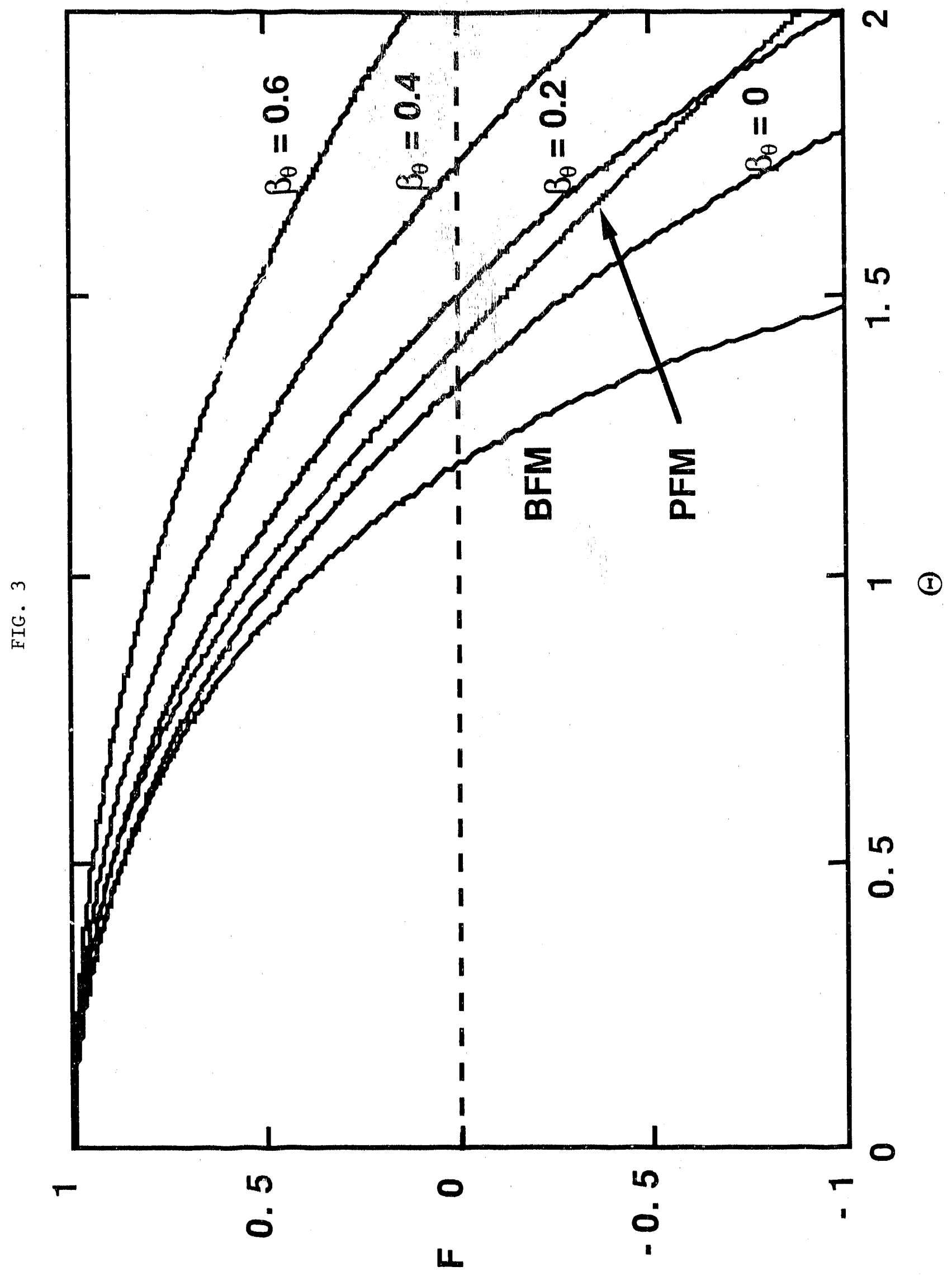









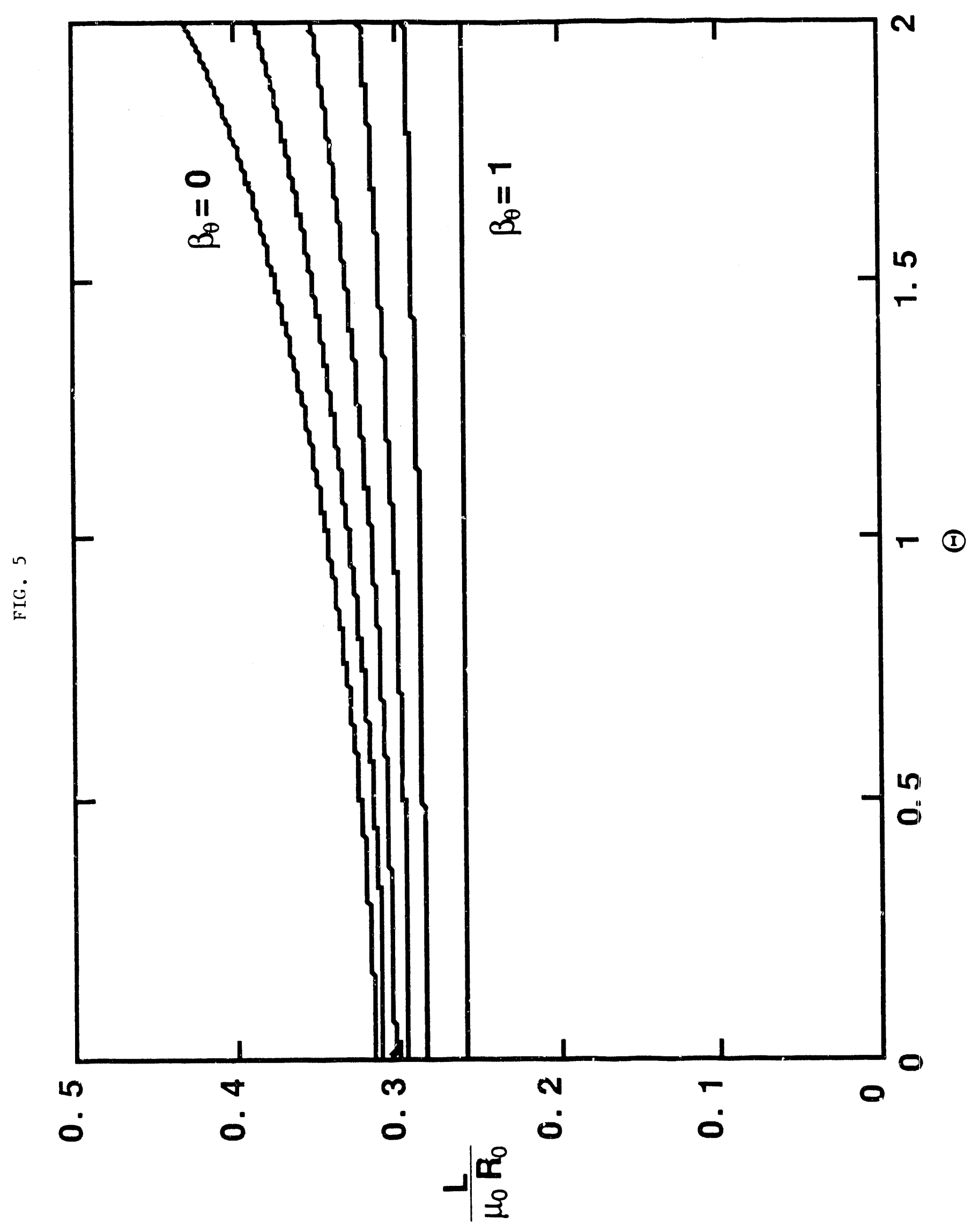




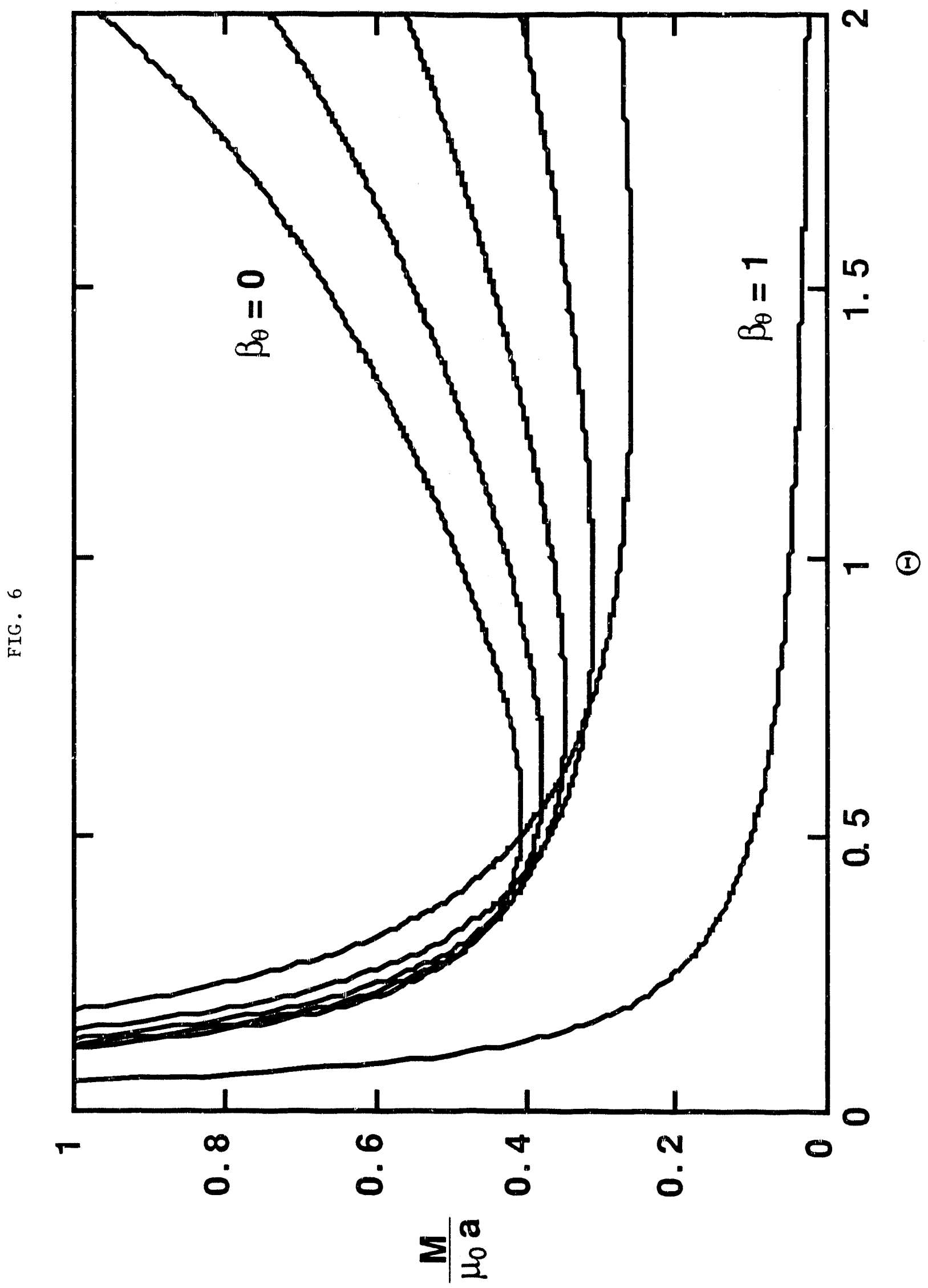




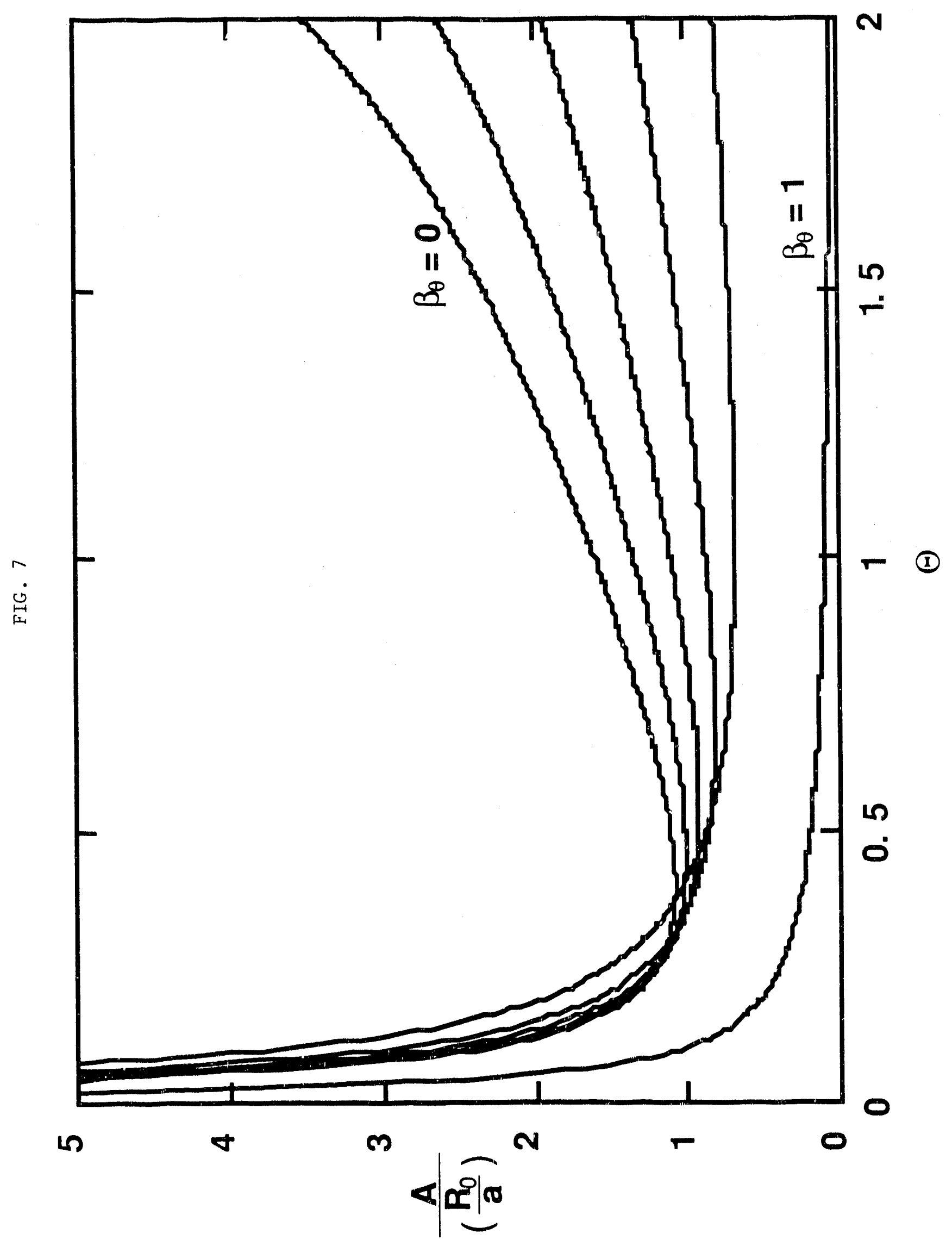









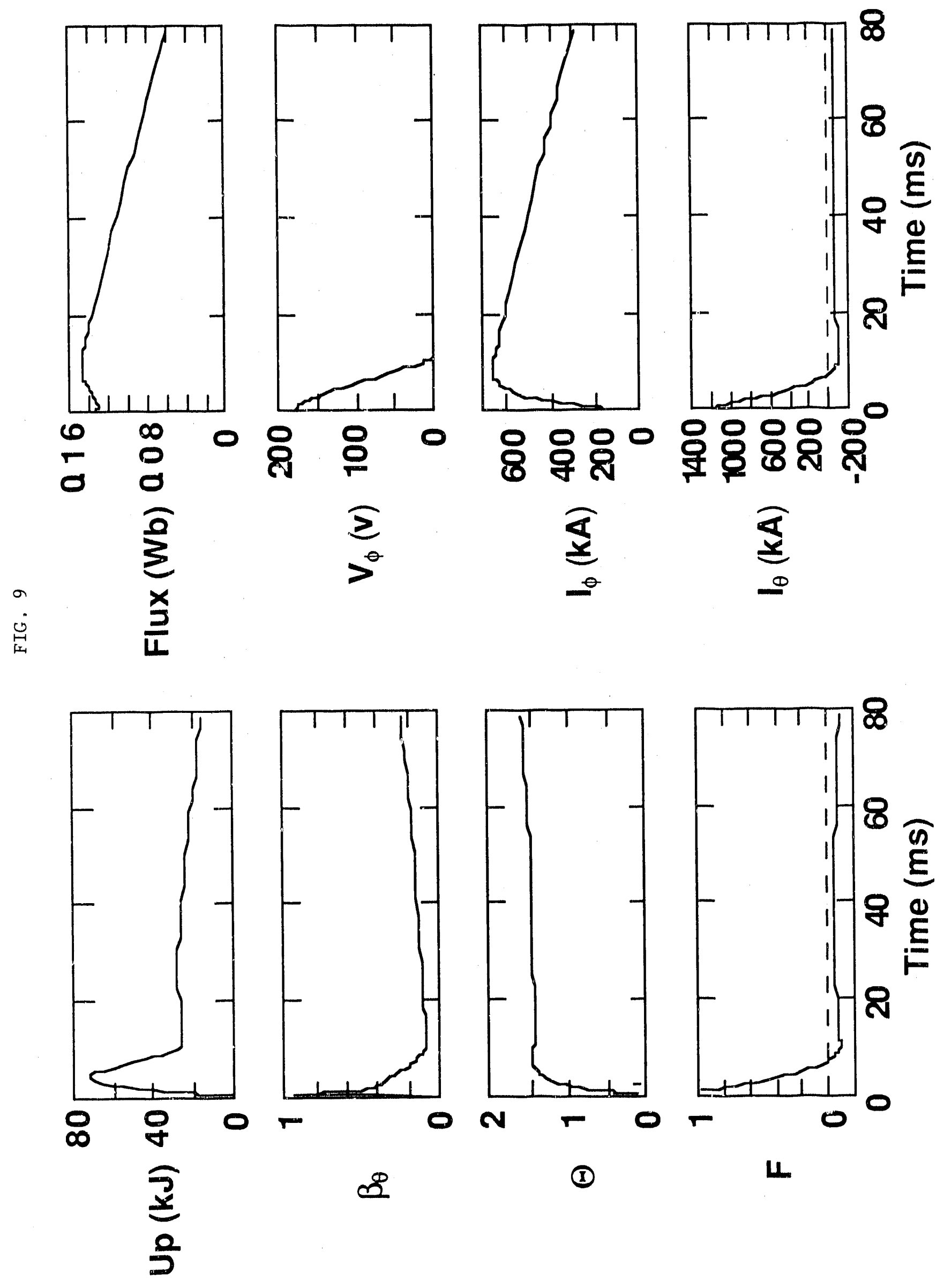



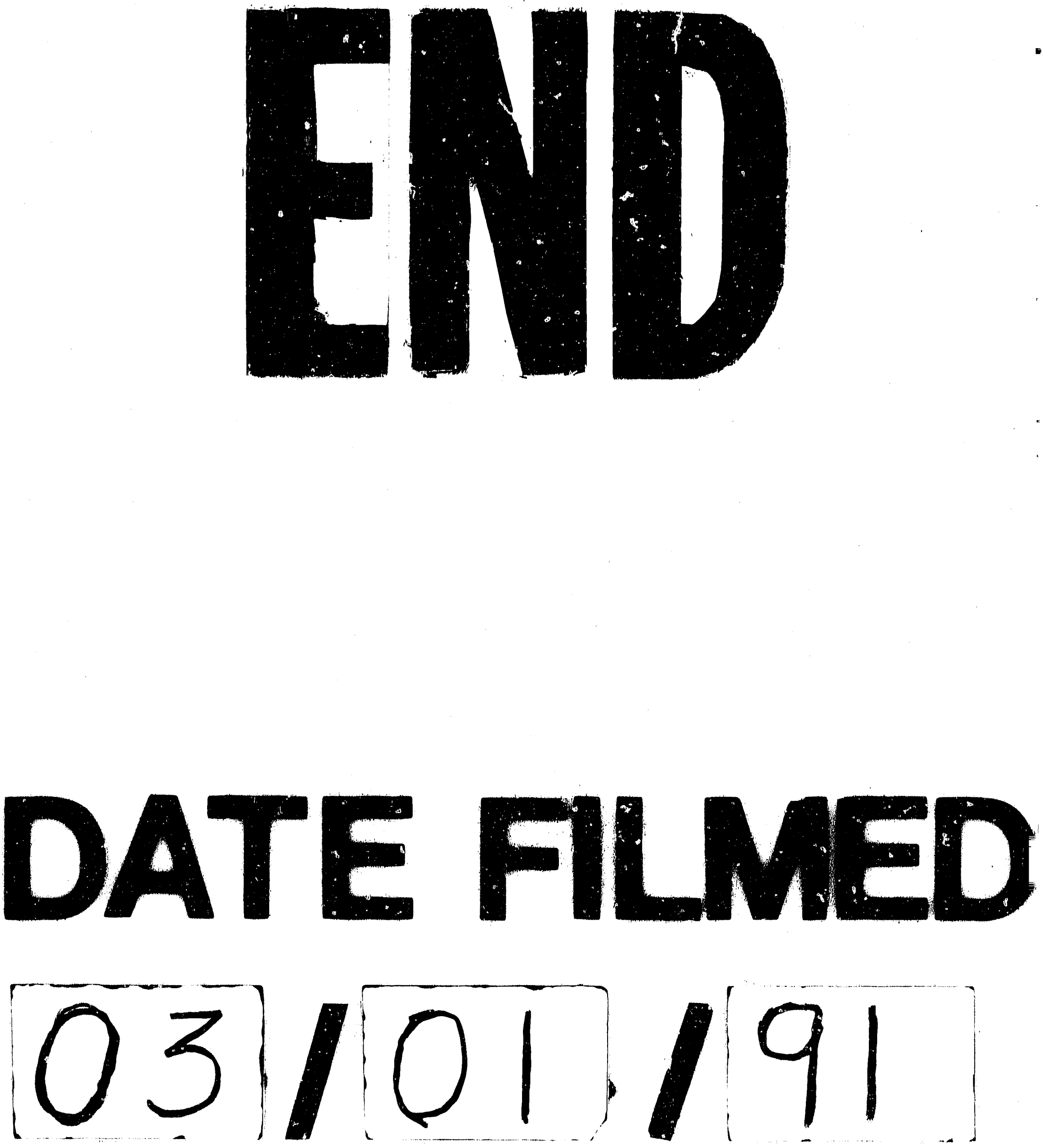
